\title{
Coherent Control of the Rotation Axis of Molecular Superrotors
}

\author{
A. Owens, ${ }^{1,2}$ A. Yachmenev, ${ }^{1,2, *}$ and J. Küpper ${ }^{1,2,3}$ \\ ${ }^{1}$ Center for Free-Electron Laser Science, Deutsches Elektronen-Synchrotron DESY, Notkestraße 85, 22607 Hamburg, Germany \\ ${ }^{2}$ The Hamburg Center for Ultrafast Imaging, Universität Hamburg, Luruper Chaussee 149, 22761 Hamburg, Germany \\ ${ }^{3}$ Department of Physics, Universität Hamburg, Luruper Chaussee 149, 22761 Hamburg, Germany
}

(Dated: July 9, 2018)

\begin{abstract}
The control of ultrafast molecular rotational motion has benefited from the development of innovative techniques in strong-field laser physics. Here, we theoretically demonstrate a novel type of coherent control by inducing rotation of an asymmetric-top molecule about two different molecular axes. An optical centrifuge is applied to the hydrogen sulfide $\left(\mathrm{H}_{2} \mathrm{~S}\right)$ molecule to create a molecular superrotor, an object performing ultrafast rotation about a well-defined axis. Using two distinct pulse envelopes for the optical centrifuge, we show that $\mathrm{H}_{2} \mathrm{~S}$ can be excited along separate pathways of rotational states. This leads to stable rotation about two entirely different molecular axes while ensuring rotation is about the propagation direction of the centrifuge, i. e., the laboratory-fixed $Z$-axis. The presented scheme to control the angular momentum alignment of a molecule will, for instance, be useful in studies of molecule-molecule or molecule-surface scattering, especially due to the large amounts of energy associated with superrotors, which can even be controlled by changing the duration of the optical centrifuge pulse.
\end{abstract}

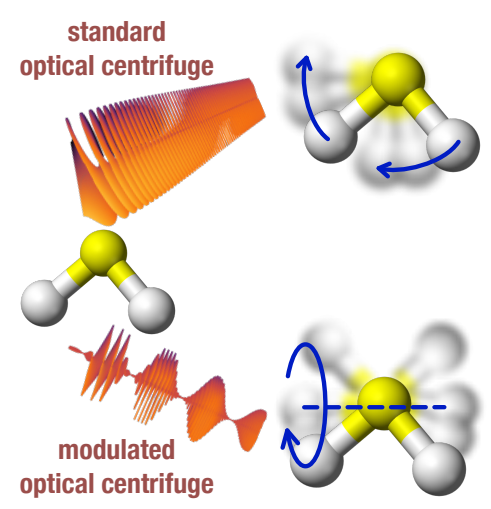

A long-standing goal of physics and chemistry is to control the motion of molecules. Tailored external fields offer a means to achieve this, ${ }^{1}$ from alignment and orientation where molecules can be spatially fixed in the laboratory frame, ${ }^{2 * 4}$ to the creation of unidirectional rotating molecular ensembles that possess oriented angular momentum. ${ }^{5 \cdots 7}$ This latter application, associated with controlled rotation, has benefited from a number of clever techniques to prepare molecules in highly excited rotational states. ${ }^{8} 9$ Perhaps the most efficient of these is the optical centrifuge, ${ }^{10} 11$ which is a strong, nonresonant, linearly-polarized laser pulse that performs accelerated rotation about the direction of propagation. The centrifuge field aligns the most polarizable molecular axis, trapping and forcing the molecule to follow the rotating laser polarization. In doing so, molecules can be adiabatically spun into extremely high angular momentum states, creating molecular "superrotors", objects with narrow, well-defined rotational wavepackets that are resistant to collisions and reorientation. ${ }^{12-14}$

The majority of studies on superrotors have involved diatomic and linear triatomic systems. Less explored are asymmetric-top molecules, which have complicated rotational spectra and thus more complex dynamics. Recently, an optical centrifuge was able to induce field-free planar alignment of $\mathrm{SO}_{2}$ with the $\mathrm{O}-\mathrm{S}-\mathrm{O}$ plane confined to the plane of the laser polarization long after the centrifuge pulse had ended. ${ }^{15}$ This demonstrated that the rotational dynamics of an asymmetric-top could be reduced to that of a simple linear rotor, which was later confirmed theoretically, ${ }^{16}$ and also highlighted the potential of the optical centrifuge as a tool for alignment and orientation. Further applications involving asymmetric-top molecules in an optical centrifuge include a novel approach to manipulate enantiomers in the gas phase through enantioselective orientation. $^{17}$

Here, we extend the possible types of rotational control over asymmetric-top molecules using nonresonant laser pulses. Our scheme is outlined in Figure 1: An optical centrifuge is applied to the hydrogen sulfide $\left(\mathrm{H}_{2} \mathrm{~S}\right)$ molecule to create a molecular superrotor. Normally, rotation takes place about the principal $c$-axis and the $\mathrm{H}-\mathrm{S}-\mathrm{H}$ plane is confined to the plane of the rotating laser polarization. Modifying the pulse envelope of the optical centrifuge, stable rotation about an entirely different molecular axis, namely the principal $a$-axis, can be obtained while still ensuring rotation is about the propagation direction of the centrifuge, i.e., the laboratory-fixed $Z$-axis. This 


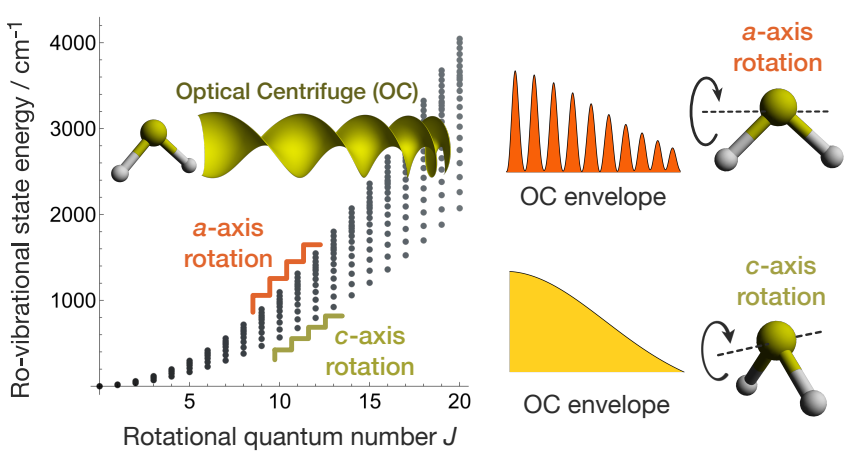

FIG. 1. Controlling the molecular axis of rotation in $\mathrm{H}_{2} \mathrm{~S}$ superrotors using an optical centrifuge. A Gaussian pulse envelope produces rotation about the principal $c$-axis and confinement of the $\mathrm{H}-\mathrm{S}-\mathrm{H}$ plane to the laboratory-fixed $X Y$ plane of the laser polarization. Using an optimized sinc function combined with a Gaussian profile for the pulse envelope, the molecule can be excited along a different pathway of rotational states leading to rotation about the principal $a$-axis.

is achieved by exciting the molecule through a different pathway of rotational states, illustrating a novel type of coherent control involving ultrafast rotation. Schemes to change the angular momentum alignment of a molecule have been demonstrated, for example, to alter the outcome of $\mathrm{H}_{2}$ molecule-surface collisions. ${ }^{18}$ Our results show that such stereodynamics studies are possible for asymmetrictop molecular superrotors, which possess large amounts of energy that, furthermore, can be controlled by adjusting the duration of the centrifuge pulse.

To realize the scheme in Figure 1, we utilize a robust, quantum mechanical approach that treats all major electronic, nuclear motion, and external field effects with high accuracy. Calculations are geared toward experimentally realizable conditions, e. g., laser parameters and timescales. The stationary rovibrational energies and eigenfunctions of $\mathrm{H}_{2} \mathrm{~S}$ up to $J=40$, where $J$ is the quantum number of the total angular momentum operator, were computed from direct numerical variational calculations ${ }^{19 \cdot 21}$ with a highly accurate, three-dimensional potential energy surface. ${ }^{22}$ External field effects, i. e., the matrix elements of the electric polarizability tensor, were evaluated in the basis of rovibrational states. The ab initio polarizability surface was generated using the coupled cluster method, CCSD (coupled cluster with all single and double excitations), with the augmented correlationconsistent triple-zeta basis set, aug-cc-pVTZ $(+\mathrm{d}$ for $\mathrm{S})$ in the frozen core approximation, and then fitted with a suitable, symmetrized analytic representation. Electronic structure calculations employed the quantum chemistry package DALTON. ${ }^{23}$ For the quantum dynamics simulations, which used the computer program RichMol, ${ }^{24}$ the time-dependent wavefunction was built from a superposition of field-free rovibrational states and the timedependent coefficients determined by numerical solution of the time-dependent Schrödinger equation using the split-operator method.
The electric field of the optical centrifuge is highly oscillating and off-resonant, therefore only the polarizability term is required in the field interaction potential,

$$
V(t)=-\frac{1}{2} \sum_{A, B=X, Y} \epsilon_{A}^{\mathrm{oc}}(t) \epsilon_{B}^{\mathrm{oc}}(t) \alpha_{A B}
$$

where $\alpha_{A B}$ are the components of the effective electric polarizability in the laboratory-fixed $X Y Z$-axis system. The optical centrifuge $\boldsymbol{\epsilon}^{\mathrm{oc}}(t)$ was applied along the laboratoryfixed $Z$-axis for a duration of $t=300$ ps and was represented by the expression,

$$
\boldsymbol{\epsilon}^{\mathrm{oc}}(t)=f(t) \epsilon_{0} \cos (\omega t)\left\{\mathbf{e}_{X} \cos \left(\beta t^{2}\right) ; \mathbf{e}_{Y} \sin \left(\beta t^{2}\right)\right\}
$$

with the peak amplitude of the field $\epsilon_{0}=8.6 \times 10^{13} \mathrm{~W} / \mathrm{cm}^{2}$, the acceleration of circular rotation $\beta=(2 \pi c)^{2} \cdot 4 \mathrm{~cm}^{-2}$, the carrier frequency of the field $\omega=c /(2 \pi \cdot 800 \mathrm{~nm})$, and the pulse envelope function

$$
f(t)=\exp \left(-t^{2} / 2 \sigma^{2}\right)
$$

where $\sigma=140$ ps. $^{17}$ Simulations were started in the rovibrational ground state $(J=k=m=0)$ which is valid for temperatures of $T \leq 3 \mathrm{~K}$. The quantum numbers $k$ and $m$ correspond to the projections, in units of $\hbar$, of $J$ onto the molecule-fixed $z$-axis and laboratory-fixed $Z$-axis, respectively. Low temperatures are necessary in asymmetric-top molecules to ensure a narrow distribution of states in the initial wavepacket and, consequently, stable rotational dynamics.

The time evolution of the external electric field for a Gaussian pulse envelope and the corresponding wavepacket populations of $\mathrm{H}_{2} \mathrm{~S}$ are plotted in Figure 2 a and $\mathrm{c}$, along with the rotational probability density function $P(\theta, \chi)=\int \mathrm{d} V \mathrm{~d} \phi \psi(t)^{*} \psi(t) \sin \theta$ at the end of the centrifuge pulse in Figure 2 e. The probability density function illustrates the orientation of the molecule with respect to the possible axes of rotation, where the Euler angles are denoted $\theta, \chi, \phi$, the volume element $\mathrm{d} V$ is associated with the three vibrational degrees of freedom, and $\psi(t)$ is the wavepacket. $\mathrm{H}_{2} \mathrm{~S}$ is primarily excited through $\Delta J=2, \Delta m=-2$ rotational Raman transitions and proceeds along a pathway of lowest energy rotational states within each $J$, i. e., those corresponding to rotation about the molecular $c$-axis. At $t=102 \mathrm{ps}$, the wavepacket is dominated by the $J=16, m=-16$ state at $1360 \mathrm{~cm}^{-1}$, but the maximum single-state population starts to rapidly decline after this time. This is due to the decreasing intensity of the centrifuge field, suggesting that the optical centrifuge pulse should be truncated between $t \approx 100-120$ ps to ensure $\mathrm{H}_{2} \mathrm{~S}$ is efficiently released in a high $J$ state. The rotational density plot shows two clear "islands", which correspond to rotation about the principal $c$-axis in either a clockwise or anticlockwise direction. The $\mathrm{H}-\mathrm{S}-\mathrm{H}$ plane is confined to the $X Y$ plane of the laser polarization which is consistent with previous studies of $\mathrm{SO}_{2}$ in an optical centrifuge. ${ }^{15} 16$ 
a) $\epsilon_{X}^{\mathrm{oc}}(t) \times 10^{10} / \mathrm{Vm}^{-1}$

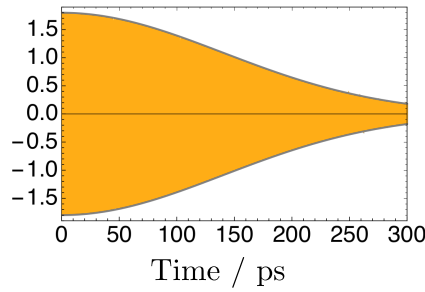

C) Population $\left|c_{i}(t)\right|^{2}$
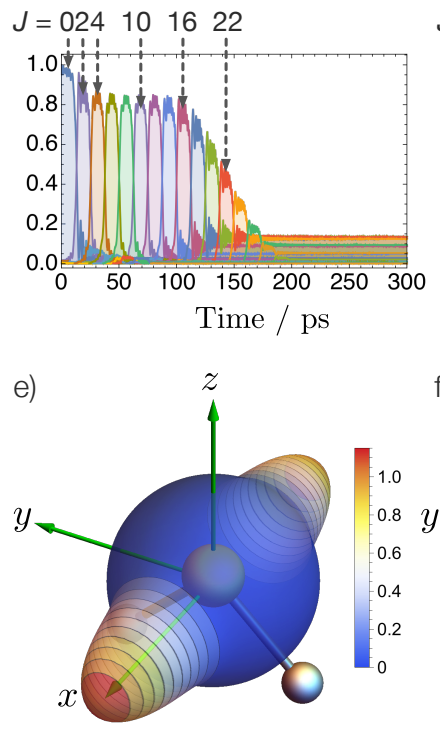

b) $\epsilon_{X}^{\mathrm{oc}}(t) \times 10^{10} / \mathrm{Vm}^{-1}$

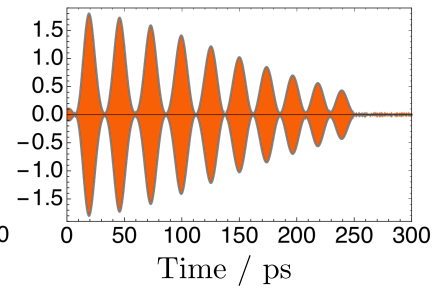

d) Population $\left|c_{i}(t)\right|^{2}$

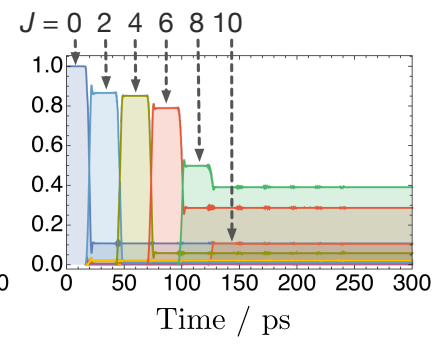

f)

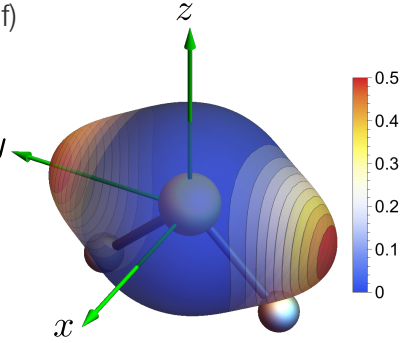

FIG. 2. Wavepacket population $\left|c_{i}(t)\right|^{2}$ and rotational probability density function $P(\theta, \chi)$ of $\mathrm{H}_{2} \mathrm{~S}$ in an optical centrifuge with a pulse envelope modeled by a Gaussian function (left panels), or with an optimized sinc function combined with a Gaussian (right panels). The $X$-projection of the electric field is shown at the top, the wavepacket population as a function of time in the middle, and $P(\theta, \chi, t=290 \mathrm{ps})$ at the bottom; $x, y, z$ refer to the molecule-fixed axis system.

To change the axis of rotation, $\mathrm{H}_{2} \mathrm{~S}$ has to be excited along a different pathway of rotational states. This can be achieved using an optical centrifuge with a modified pulse envelope such that the field intensity is at a maximum only when the angular frequency of the centrifuge is in resonance with the desired transition frequency between two states, thus enabling population transfer. The intensity is then minimized as the angular frequency increases and sweeps through undesired transitions before being maximized again at a later resonant frequency. The time points $t_{i}^{\text {res }}$ at which the angular frequency of the optical centrifuge in (2) reaches resonance with the desired rotational transition with frequency $\nu_{i}^{\text {res }}$ can be evaluated as $t_{i}^{\text {res }}=\nu_{i}^{\text {res }} /(4 \beta)$. Now, to force $\mathrm{H}_{2} \mathrm{~S}$ to rotate about the principal $a$-axis the rotational excitation must be guided through the upper energy rotational state within each $(2 J+1)$ multiplet. The respective $J \rightarrow J+2$ transition wavenumbers are $58.4,138.4$, $220.0,300.1$, and $377.6 \mathrm{~cm}^{-1}$, corresponding to frequen- cies of $1750.8,4149.1,6595.4,8996.7$, and $11320.1 \mathrm{GHz}$, for $J=0,2,4,6,8$, which result in resonant times of $t_{i}^{\text {res }}=19.4,45.9,73.0,99.6,125.3 \mathrm{ps}$, respectively, for the centrifuge acceleration parameter $\beta=(2 \pi c)^{2} \cdot 4 \mathrm{~cm}^{-2}$. The pulse profile function is modeled by a sum of sinc functions centered at the different resonant times $t_{i}^{\text {res }}$ weighted with a Gaussian envelope function, that is

$$
f(t)=\sum_{i}\left(\frac{\sin \left(\left(t-t_{i}^{\mathrm{res}}\right) / \sigma_{s}\right)}{\left(t-t_{i}^{\mathrm{res}}\right) / \sigma_{s}}\right)^{2} \exp \left(-t^{2} / 2 \sigma^{2}\right),
$$

where $\sigma_{s}=4 \mathrm{ps}$ and $\sigma=140 \mathrm{ps}$. The resulting pulse profile function is plotted in Figure $2 \mathrm{~b}$ together with the results of the simulation in Figure $2 \mathrm{~d}$ and $\mathrm{f}$. At $t=102 \mathrm{ps}$, the wavepacket is dominated by the $J=8, m=-8$ rotational state at $717 \mathrm{~cm}^{-1}$, which corresponds to rotation about the principal $a$-axis, indicated by the two shifted islands on the probability density plot. Since this is a lower $J$ state, the rotational density is broader and not as pronounced as in the first scenario. Because $m=-J$, the axis of rotation is along the laboratory-fixed $Z$-axis, i. e., the propagation direction of the optical centrifuge. Essentially, the molecule has assumed a perpendicular orientation compared to the case of the standard centrifuge excitation shown in Figure 2 e. This can be analyzed by looking at the quantum numbers $k_{a}$ and $k_{c}$ corresponding to the projection of the total angular momentum onto the molecule-fixed $a$ and $c$-axis, respectively. For standard centrifuge excitation $k_{c} \approx J$, while for the modified pulse envelope excitation $k_{a} \approx J$. Note that the sinc function pulse envelope could also be optimized for standard centrifuge-type excitation, but this does not yield any major improvements in population transfer.

Since $\mathrm{H}_{2} \mathrm{~S}$ is a relatively light, fast rotor, efficient rotational excitation to much higher $J$ would require centrifuge pulses of several-hundred-picosecond duration. ${ }^{25}$ Interestingly, $\mathrm{H}_{2} \mathrm{~S}$ displays rotational energy level clustering for large $J,{ }^{26} 27$ a characteristic effect of polyatomic molecules with local mode vibrations. ${ }^{28}$ After a certain critical $J$ value, which for $\mathrm{H}_{2} \mathrm{~S}$ is around $J_{c} \approx 15$, the rotational energy levels form fourfold degenerate clusters as shown in Figure 3 for the ground vibrational state. Assuming population can be transferred along the correct pathway of rotational states, as $\mathrm{H}_{2} \mathrm{~S}$ climbs the rotational ladder from $J \approx 16$ to $J \approx 30$, the axis of rotation gradually moves from the principal $a$-axis to an axis coinciding with one of the $\mathrm{S}-\mathrm{H}$ bonds, as shown in the probability density plot of Figure 3. These so-called cluster states also have the unique property of being chiral, ${ }^{29}$ where rotation in either a clockwise or anticlockwise direction corresponds to the two rotating enantiomers of a dynamically chiral system. ${ }^{30}$ However, while it could offer intriguing opportunities for controlling chirality, clustering is not a necessary condition for the coherent control method proposed here, which is applicable to molecules of arbitrary structure.

In conclusion, we have shown that asymmetric-top molecules can be made to rotate in a stable manner 


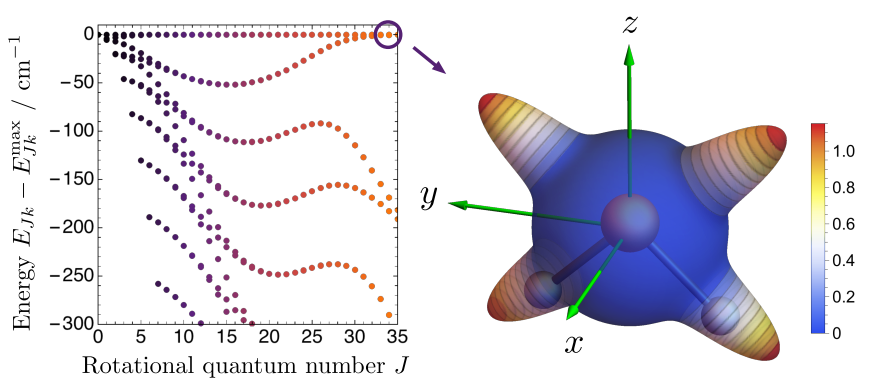

FIG. 3. Rotational energy level clustering in the ground vibrational state of $\mathrm{H}_{2} \mathrm{~S}$. In the left panel, the energy difference $E_{J k}-E_{J k}^{\max }$ has been plotted for each rotational state, with energy $E_{J k}$, relative to the maximum energy $E_{J k}^{\max }$ in its $J$ multiplet. As $J$ increases, after a certain critical $J$ value the energy difference between energy levels starts to decrease and rotational cluster states form. ${ }^{26}{ }^{27}$ In the right panel, the rotational probability density function $P(\theta, \chi)$ is illustrated for the top cluster state at $J=35$, which shows four possible axes of rotation coinciding with the $\mathrm{S}-\mathrm{H}$ bonds. Here, $x y z$ refers to the molecule-fixed axis system.

about two entirely different molecular axes. This was achieved for $\mathrm{H}_{2} \mathrm{~S}$ using optical centrifuges with different pulse envelopes, tailored to excite the molecule though distinct pathways of rotational states. The proposed scheme is relatively robust and there is some tolerance for shot-to-shot fluctuations in the chirp rate $\beta$ and the sinc function width parameter $\sigma_{s}$, e.g., at least $\pm 1 \%$ for the values used in this study. Furthermore, for larger values of $\sigma_{s}$ and/or smaller values of $\beta$, the robustness against such fluctuations will increase. Lower field intensities, although resulting in less efficient rotational excitation and a reduced population of the final target state, can also be used to avoid potential ionization. Perhaps the most challenging aspect of the proposed scheme is the sinc function pulse envelope, however, the ability to shape optical pulses is a rapidly developing field of research. ${ }^{31}$

Our simulations utilized laser parameters well within the current capabilities of laser technology, with calculations based on robust, first principles methodologies geared toward high accuracy, which provide detailed information on the energy level structure and electric tensor transition moment matrix elements. More sophisticated coherent control schemes could thus be developed to prepare molecules of arbitrary structure in specific, highly excited rotational states. Extending this approach to larger or heavier systems will pose challenges, most notably the increased density of rotational states at lower energies, which makes it easier to populate undesired energy levels and select the wrong rotational pathway, producing "chaotic" dynamics; this can be mitigated using state-selection before applying the control pulses. ${ }^{32}$ Despite these difficulties, the ability to force stable rotation about different molecular axes and to change the angular momentum alignment of a molecule would be highly advantageous in molecule-molecule or molecule-surface scattering experiments, particularly with superrotors which display novel properties and contain large amounts of rotational energy.

\section{ACKNOWLEDGMENTS}

This work has been supported by the Deutsche Forschungsgemeinschaft (DFG) through the excellence cluster "The Hamburg Center for Ultrafast Imaging Structure, Dynamics and Control of Matter at the Atomic Scale" (CUI, EXC1074) and the priority program 1840 "Quantum Dynamics in Tailored Intense Fields" (QUTIF, KU1527/3), by the European Research Council under the European Union's Seventh Framework Programme (FP7/2007-2013) through the Consolidator Grant COMOTION (ERC-614507-Küpper), by the Helmholtz Gemeinschaft through the "Impuls- und Vernetzungsfond", and by the COST action MOLIM (CM1405).

\section{REFERENCES}

*andrey.yachmenev@cfel.de; https://www.controlledmolecule-imaging.org

(1) Lemeshko, M.; Krems, R. V.; Doyle, J. M.; Kais, S. Manipulation of molecules with electromagnetic fields. Mol. Phys. 2013, 111, 1648-1682.

(2) Stapelfeldt, H.; Seideman, T. Colloquium: Aligning molecules with strong laser pulses. Rev. Mod. Phys. 2003, $75,543-557$.

(3) Holmegaard, L.; Nielsen, J. H.; Nevo, I.; Stapelfeldt, H.; Filsinger, F.; Küpper, J.; Meijer, G. Laser-induced alignment and orientation of quantum-state-selected large molecules. Phys. Rev. Lett. 2009, 102, 023001.

(4) Ghafur, O.; Rouzée, A.; Gijsbertsen, A.; Siu, W. K.; Stolte, S.; Vrakking, M. J. J. Impulsive orientation and alignment of quantum-state-selected NO molecules. Nat. Phys. 2009, 5, 289-293.

(5) Fleischer, S.; Khodorkovsky, Y.; Prior, Y.; Averbukh, I. S. Controlling the sense of molecular rotation. New J. Phys. 2009, 11, 105039.

(6) Kitano, K.; Hasegawa, H.; Ohshima, Y. Ultrafast angular momentum orientation by linearly polarized laser fields. Phys. Rev. Lett. 2009, 103, 223002.

(7) Zhdanovich, S.; Milner, A. A.; Bloomquist, C.; Floß, J.; Sh. Averbukh, I.; Hepburn, J. W.; Milner, V. Control of molecular rotation with a chiral train of ultrashort pulses. Phys. Rev. Lett. 2011, 107, 243004.

(8) Ohshima, Y.; Hasegawa, H. Coherent rotational excitation by intense nonresonant laser fields. Int. Rev. Phys. Chem. 2010, 29, 619-663.

(9) Milner, V.; Hepburn, J. W. Laser control of ultrafast molecular rotation. Adv. Chem. Phys. 2016, 159, 395412 .

(10) Karczmarek, J.; Wright, J.; Corkum, P.; Ivanov, M. Optical centrifuge for molecules. Phys. Rev. Lett. 1999, $82,3420-3423$.

(11) Villeneuve, D. M.; Aseyev, S. A.; Dietrich, P.; Spanner, M.; Ivanov, M. Y.; Corkum, P. B. Forced molecular rotation in an optical centrifuge. Phys. Rev. Lett. 2000, $85,542-545$

(12) Yuan, L.; Teitelbaum, S. W.; Robinson, A.; Mullin, A. S. Dynamics of molecules in extreme rotational states. Proc. Natl. Acad. Sci. U. S. A. 2011, 108, 6872-6877. 
(13) Milner, A. A.; Korobenko, A.; Hepburn, J. W.; Milner, V. Effects of ultrafast molecular rotation on collisional decoherence. Phys. Rev. Lett. 2014, 113, 043005.

(14) Khodorkovsky, Y.; Steinitz, U.; Hartmann, J.-M.; Sh. Averbukh, I. Collisional dynamics in a gas of molecular super-rotors. Nat. Commun. 2015, 6, 7791.

(15) Korobenko, A.; Milner, V. Adiabatic field-free alignment of asymmetric top molecules with an optical centrifuge. Phys. Rev. Lett. 2016, 116, 183001.

(16) Omiste, J. J. Theoretical study of asymmetric superrotors: Alignment and orientation. Phys. Rev. A 2018, 97, 023407.

(17) Tutunnikov, I.; Gershnabel, E.; Gold, S.; Sh. Averbukh, I. Selective orientation of chiral molecules by laser fields with twisted polarization. J. Phys. Chem. Lett. 2018, 9, 1105-1111.

(18) Godsi, O.; Corem, G.; Alkoby, Y.; Cantin, J. T.; Krems, R. V.; Somers, M. F.; Meyer, J.; Kroes, G.-J.; Maniv, T.; Alexandrowicz, G. A general method for controlling and resolving rotational orientation of molecules in molecule-surface collisions. Nat. Commun. 2017, 8, 15357.

(19) Yurchenko, S. N.; Thiel, W.; Jensen, P. Theoretical ROVibrational energies (TROVE): A robust numerical approach to the calculation of rovibrational energies for polyatomic molecules. J. Mol. Spectrosc. 2007, 245, 126 140.

(20) Yachmenev, A.; Yurchenko, S. N. Automatic differentiation method for numerical construction of the rotationalvibrational Hamiltonian as a power series in the curvilinear internal coordinates using the Eckart frame. J. Chem. Phys. 2015, 143, 014105.

(21) Yurchenko, S. N.; Yachmenev, A.; Ovsyannikov, R. I. Symmetry adapted ro-vibrational basis functions for vari- ational nuclear motion calculations: TROVE approach J. Chem. Theory Comput. 2017, 13, 4368.

(22) Azzam, A. A. A.; Tennyson, J.; Yurchenko, S. N.; Naumenko, O. V. ExoMol molecular line lists - XVI. the rotation-vibration spectrum of hot $\mathrm{H}_{2}$ S. Mon. Not. R. Astron. Soc. 2016, 460, 4063-4074.

(23) Aidas, K. et al. The Dalton quantum chemistry program system. WIREs Comput. Mol. Sci. 2014, 4, 269-284.

(24) Owens, A.; Yachmenev, A. RichMol: A general variational approach for rovibrational molecular dynamics in external electric fields. J. Chem. Phys. 2018, 148, 124102.

(25) Trippel, S.; Mullins, T.; Müller, N. L. M.; Kienitz, J. S.; Długołęcki, K.; Küpper, J. Strongly aligned and oriented molecular samples at a $\mathrm{kHz}$ repetition rate. Mol. Phys. 2013, 111, 1738.

(26) Pyka, J. Clustering of rovibrational energy levels in the highly excited $\mathrm{H}_{2} \mathrm{~S}$ molecule. Mol. Phys. 1990, 70, 547561.

(27) Kozin, I. N.; Jensen, P. Fourfold clusters of rovibrational energy levels for $\mathrm{H}_{2} \mathrm{~S}$ studied with a potential energy surface derived from experiment. J. Mol. Spectrosc. 1994, 163, 483-509.

(28) Jensen, P. Local modes in vibration-rotation spectroscopy. WIREs Comput. Mol. Sci. 2012, 2, 494-512.

(29) Bunker, P. R.; Jensen, P. Chirality in rotational energy level clusters. J. Mol. Spectrosc. 2004, 228, 640-644.

(30) Owens, A.; Yachmenev, A.; Yurchenko, S. N.; Küpper, J. Climbing the rotational ladder to chirality. submitted 2018, arXiv:1802.07803 [physics.chem-ph].

(31) Goswami, D. Optical pulse shaping approaches to coherent control. Phys. Rep. 2002, 374, 385-481.

(32) Chang, Y.-P.; Horke, D. A.; Trippel, S.; Küpper, J. Spatially-controlled complex molecules and their applications. Int. Rev. Phys. Chem. 2015, 34, 557-590. 